\title{
Lymphonodal metastases in incidental thyroid microcarcinomas verified by sentinel lymph node biopsy of jugulo-carotid chain
}

\begin{abstract}
Authors: Gavrilovic ${ }^{4}$, Radan Dzodic ${ }^{1,2}$

1 Institute for Oncology and Radiology of Serbia, Surgical Oncology Clinic, Pasterova 14, Belgrade, Serbia

2 University of Belgrade, School of Medicine, Dr Subotica 8, Belgrade, Serbia

${ }^{3}$ Institute for Oncology and Radiology of Serbia, Department of Pathology, Pasterova 14, Belgrade, Serbia

${ }^{4}$ Institute for Oncology and Radiology of Serbia, Data Center, Pasterova 14, Belgrade, Serbia
\end{abstract}

Merima Orucil, Ivan Markovic l,2, Nada Santracl', Igor Djurisicl, Marko Butal', Gordana Pupic ${ }^{3}$, Dusica
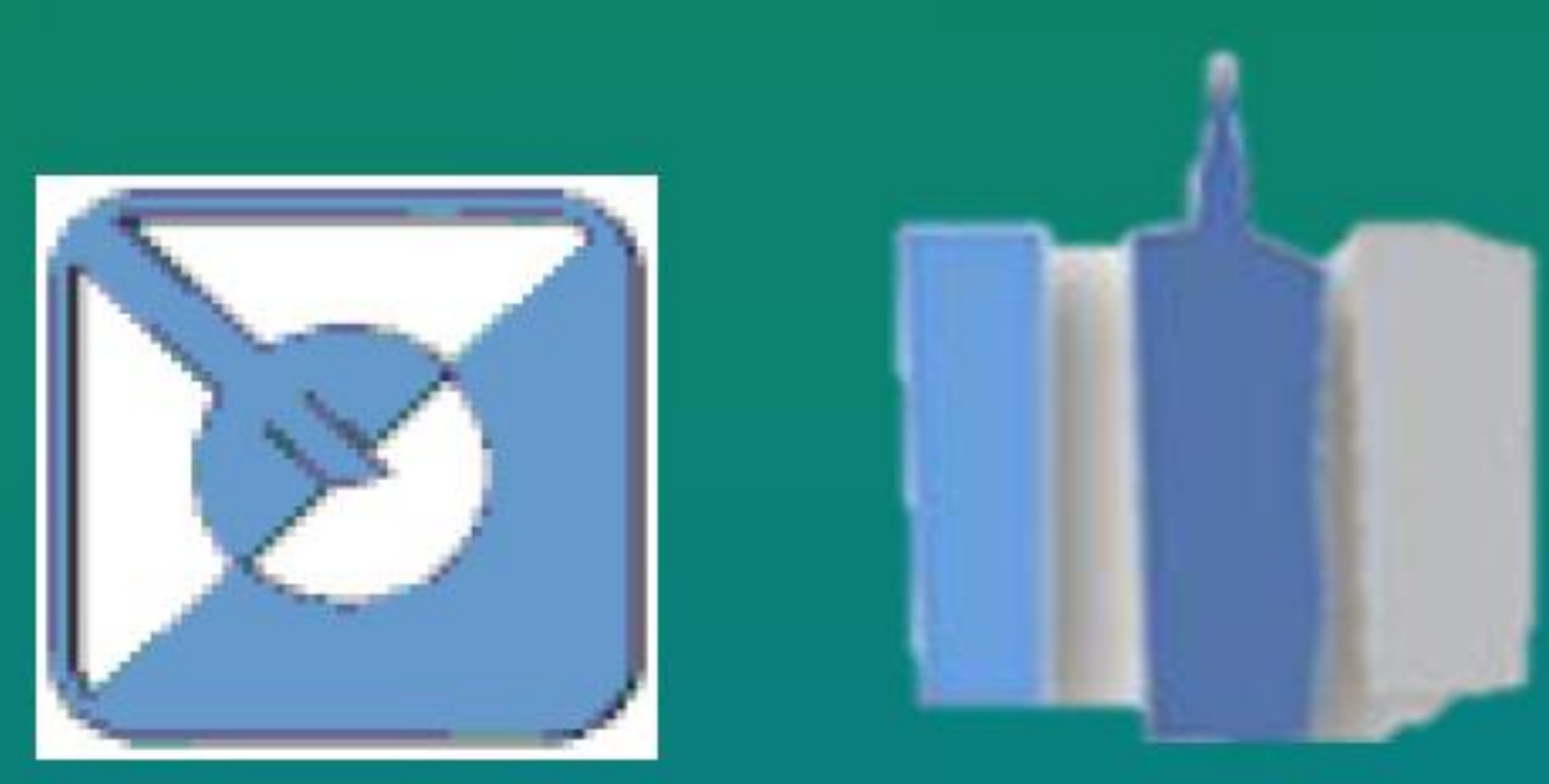

\section{INTRODUCTION}

Thyroid cancers $\leq 1 \mathrm{~cm}$ in size are defined as microcarcinomas [1]. The aim of this study was to determine frequency of lymphonodal metastases in jugulo-carotid chain (JCC) and central neck compartment in incidental thyroid microcarcinomas (ITMC), as well as to examine whether sentinel lymph node (SLN) biopsy of JCC is an accurate technique to select patients with true positive, but clinically and ultrasonically negative lymph nodes, for modified radical neck dissection (MRND).

\section{MATERIALS AND METHODS}

Out of all patients operated in our Institution from 2004 to 2013 for multinodal goitre, Hashimoto's thyroiditis, adenomas, Graves' disease or increase of serum calcitonin concentration, for the purpose of this study, we have selected 117 who had ITMC and were surgically treated with total thyroidectomy (lobectomy in only one patient), sampling or complete dissection of central lymph nodes and SLN biopsy in JCC. As a standard procedure, SLN mapping was performed with $0.2 \mathrm{ml}$ of $1 \%$ methylene blue dye injected just beneath thyroid gland capsule. SLNs were examined by frozen section and if positive, additional MRND was done. Hystopathological analysis showed 108 papillary, 6 medullary, and 3 combined papillary and medullary thyroid microcarcinomas less than $1 \mathrm{~cm}$ in diameter.

\section{RESULTS}

Multicentric carcinomas were recorded in $39.32 \%$ of patients. In patients with lymphonodal metastases $(24.79 \%)$, JCC metastases were found in $7.69 \%$, central in $20.51 \%$.

Specificity and sensitivity of method are $100 \%$ and $57.14 \%$, positive and negative predictive values are $100 \%$ and $97.3 \%$. Method's accuracy is $97.39 \%$.

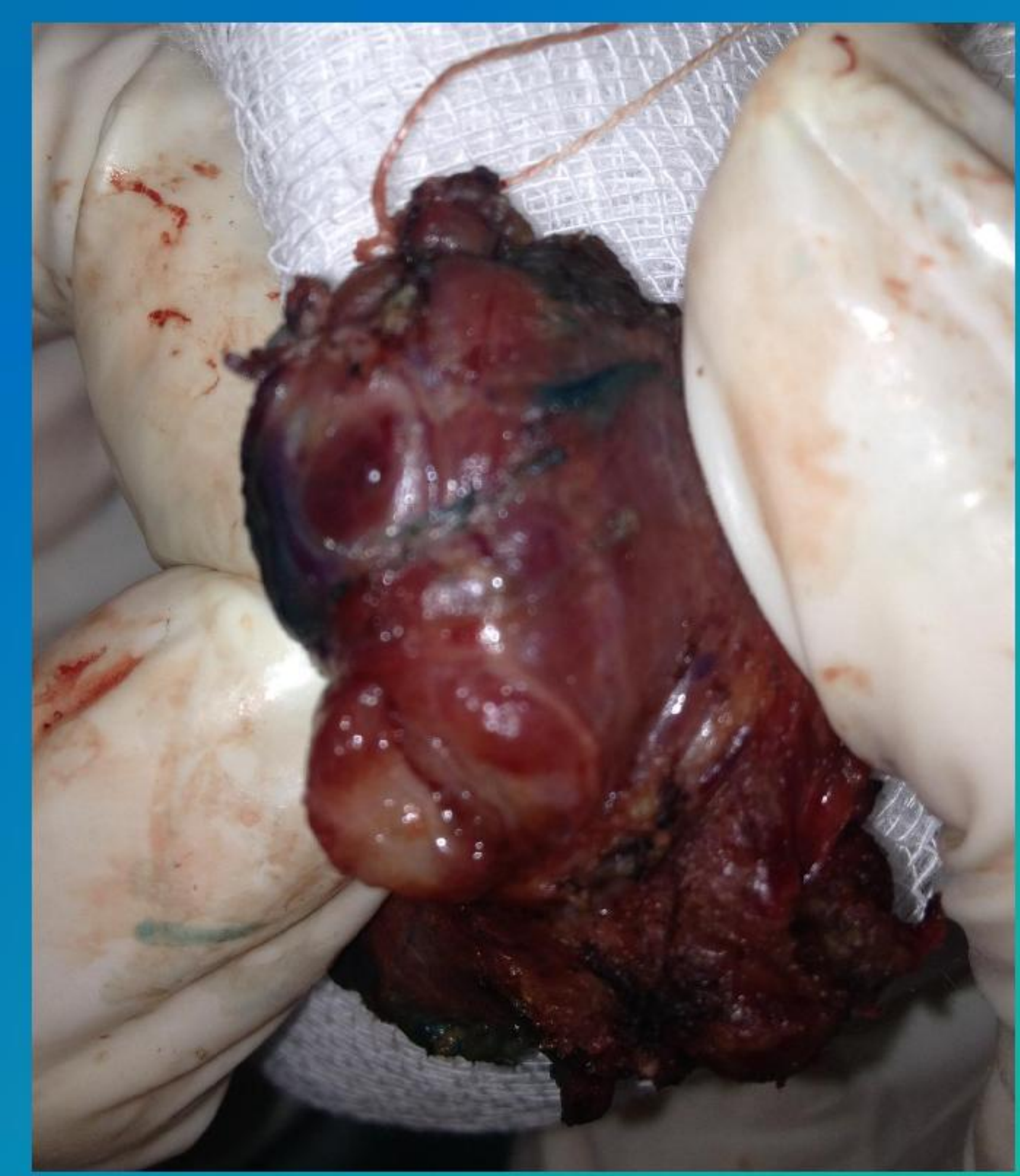

Figure: Macroscopic appearance of thyroid microcarcinoma in the right lobe

\section{CONCLUSION}

Data showed high percentage of metastases in central neck compartment. SLN biopsy is a method more precise than clinical examination and ultrasonography in detection of ITMC lateral lymphonodal metastases in NO patients. Using SLN biopsy for intraoperative assessment of lateral compartment one can avoid unnecessary MRND, as well as prevent under-treatment of patients with good prognosis. In addition, this method helps optimizing ablative radioiodine treatment.

\section{REFERENCES}

